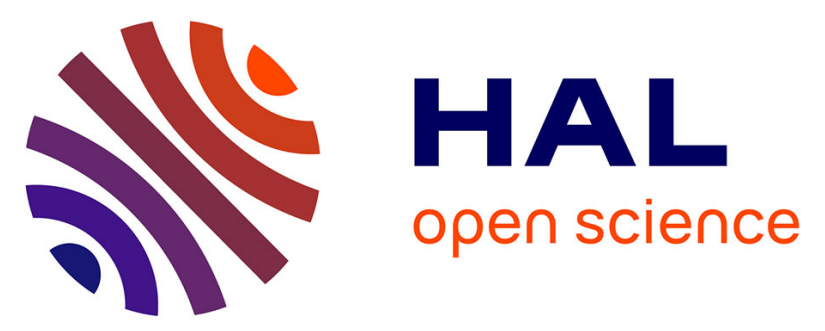

\title{
Long-term outcome of parenthood project during in vitro fertilization and after discontinuation of unsuccessful in vitro fertilization
}

Elise de La Rochebrochard, Céline Quelen, Rusudan Peikrishvili, Juliette Guibert, Jean Bouyer

\section{To cite this version:}

Elise de La Rochebrochard, Céline Quelen, Rusudan Peikrishvili, Juliette Guibert, Jean Bouyer. Long-term outcome of parenthood project during in vitro fertilization and after discontinuation of unsuccessful in vitro fertilization. Fertility and Sterility, 2009, 92 (1), pp.149-156. 10.1016/j.fertnstert.2008.05.067 . hal-02263336

\section{HAL Id: hal-02263336 \\ https://hal.science/hal-02263336}

Submitted on 4 Aug 2019

HAL is a multi-disciplinary open access archive for the deposit and dissemination of scientific research documents, whether they are published or not. The documents may come from teaching and research institutions in France or abroad, or from public or private research centers.
L'archive ouverte pluridisciplinaire HAL, est destinée au dépôt et à la diffusion de documents scientifiques de niveau recherche, publiés ou non, émanant des établissements d'enseignement et de recherche français ou étrangers, des laboratoires publics ou privés. 
Publisher's Version/PDF in open access

on editor web site:

http://www.fertstert.org/article/S0015-0282\%2808\%2901181-3/pdf

La Rochebrochard Elise (de), Quelen Céline, Peikrishvili Rusudan, Guibert Juliette, Bouyer Jean, 2009, "Long-term outcome of parenthood project during IVF and after discontinuation of unsuccessful IVF”, Fertility and Sterility, 92(1), p. 149-156. DOI:

10.1016/j.fertnstert.2008.05.067

\section{Long-term outcome of parenthood project}

\section{during IVF and after discontinuation of unsuccessful IVF}

Elise de La Rochebrochard, Ph.D, a b c Céline Quelen, M.Sc., a b c Rusudan

Peikrishvili, M.D., d Juliette Guibert, M.D., e Jean Bouyer, Ph.D. a b c

a INSERM Unit 822, Le Kremlin-Bicêtre, F-94276, France

b INED, F-94276 Le Kremlin-Bicêtre, France

c Université Paris-Sud 11, Faculté de Médecine Paris-Sud, Hôpital de Bicêtre, F94276 Le Kremlin-Bicêtre, France

d Unité de FIV, CHU de Clermont Ferrand, F-63003 Clermont Ferrand, France 
e Unité de Médecine de la Reproduction, Service de Gynécologie-Obstétrique II, Hôpital Cochin, F-75014 Paris, France

CORRESPONDING AUTHOR: Elise de La Rochebrochard, INSERM-INED U822, Porte 26, 82, rue du Général Leclerc, F-94276 Le Kremlin-Bicêtre Cedex, France. Phone: 33-1-45-21-23-33. Fax: 33-1-45-21-20-75. E-mail: roche@ined.fr.

Conflict of interest: none declared

Running title: Long-term outcome of IVF patients

Capsule: $66 \%$ of IVF patients may finally succeed in having a child: $40 \%$ during IVF treatment and $26 \%$ after discontinuing treatment (by adoption or spontaneous pregnancy). 


\section{ABSTRACT}

Objective: To explore the long-term outcome of patients who began IVF treatment by considering not only treatment outcome in the center but also the parenthood project outcome after discontinuation of unsuccessful IVF.

Design: Retrospective cohort follow-up study.

Setting: Two French IVF centers.

Patient(s): 724 patients who began IVF treatment in 1998.

Intervention(s): Postal and phone contacts with unsuccessful IVF patients.

Main Outcome Measure(s): Long-term outcome of parenthood project.

Result(s). Of the 724 patients, a minimum of $53 \%$ and a maximum of $81 \%$ finally succeeded in their parenthood project during or after IVF treatment (depending on the hypotheses that the 204 patients not contacted either failed or succeeded in their parenthood project). An intermediate hypothesis gave an estimation of $66 \%$ of patients finally succeeding in having a child ( $40 \%$ during IVF treatment in the center and $26 \%$ after). Achievement of the parenthood project after IVF discontinuation was mainly due to adoption of a child (46\%) or a birth following a spontaneous pregnancy $(42 \%)$.

Conclusion(s): Unsuccessful patients should not lose hope, as nearly half may subsequently succeed in having a child.

Key Words: in vitro fertilization; follow-up study; treatment outcome; pregnancy; adoption 


\section{INTRODUCTION}

Infertility is an important public health issue, as $9 \%$ of couples in developed countries do not conceive within 12 months of attempting pregnancy and it is estimated that $56 \%$ of infertile couples seek medical help (1). In response to infertility, assisted reproductive techniques (ART) have been developing rapidly in the last thirty years. In the European ART report for the year 2003, 15 countries (including Denmark, France, Germany, Norway, Sweden, UK) exhaustively reported their activity, finding a rate of 1,022 ART cycles per million inhabitants (2).

In spite of a clear increase in the number of ART cycles performed worldwide, these treatments remain an emotional and physical burden for the patient and her male partner $(3,4)$ and drop-out rates are high. In countries where the state subsidizes three IVF cycles, it has been observed that drop-out rates before the third cycle are as high as $62 \%$ in the Netherlands (5) and $65 \%$ in Sweden (6). In the United Kingdom where IVF treatment is often financed by the couples themselves, it has been observed than $64 \%$ of couples discontinued treatment after only one unsuccessful IVF cycle (7). These high drop-out rates raise several questions. The first is the reason for discontinuation. The few papers having addressed this issue highlighted the importance of predictors of failure and the psychological burden of treatment, especially when IVF cycles are state-subsidized (7-10). A second question is to how to take drop-outs into account when estimating the probability that IVF couples will finally have a child. In evaluation of the efficacy of IVF, this issue has been addressed by advancing hypotheses on the couples' probability of success if

they had continued IVF treatment $(5,6,11,12)$. Another approach would be to examine the real chance of becoming parents for patients starting IVF, either during 
the treatment itself or after drop-out. In particular, it would be of great interest to investigate whether drop-out patients finally succeed in having a child, possibly by transition of their parenthood project toward adoption.

In order to address this issue, we followed patients from the beginning of IVF treatment and examined not only treatment outcome in the center but also the longterm parenthood project outcome after discontinuation of unsuccessful treatment. 


\section{MATERIALS AND METHODS}

\section{Design and setting}

Patients were from two French IVF centers in Paris (Cochin Hospital) and in Clermont-Ferrand, a medium-sized city in central France. All patients who had their first oocyte retrieval in one of these centers in 1998 were included. The study received approval from the French Data Protection Authority (CNIL) in September 2005 (authorization number 05-1334).

In May 2006, a postal questionnaire was sent to women who had discontinued IVF treatment without achieving a live birth. Treatment discontinuation was defined as no oocyte retrieval, no fresh or frozen embryo transfer, and no IVF pregnancy in the inclusion center within two years preceding the mailing. A postal questionnaire was mailed to the address recorded at the time of treatment. If the mail was returned because the patient was not known at that address, we searched for an up-to-date address in the hospital general addresses database and in the French national phone directory. In September 2006, a second postal mail including a refusal form was sent to non-respondents. If we received no answer to the two postal mails, we tried to contact the patient by telephone between November and December 2006 in order to complete a very short phone questionnaire. 


\section{Information Collected}

Information on IVF attempts in the inclusion center was collected from medical records: infertility diagnosis, successive oocyte retrievals undergone by the patients in the center (conventional IVF or ICSI), outcomes of IVF pregnancies obtained either by fresh or frozen embryo transfers.

The postal questionnaire covered a wide range of topics: sociodemographic characteristics, fertility before 1998, treatments undergone before 1998, patient's feelings about IVF treatment, IVF discontinuation, patient's history since the end of IVF treatment in the inclusion center, in particular IVF attempts in another center, other medical treatments, spontaneous pregnancies, adoption procedures, bringing up other children, opinion on IVF treatment, consequences of IVF treatment. The short phone questionnaire was restricted to patient's profession, success in having a child after the end of IVF treatment in the inclusion center, spontaneous pregnancies, and psychological consequences of IVF treatment.

\section{Statistical Methods}

Data were analyzed using SAS software (9.0 version). Chi-square tests or Fisher exact tests were used for comparing percentages. Crude and adjusted odds ratios $(\mathrm{OR})$ and $95 \%$ confidence intervals $(\mathrm{Cl})$ were estimated by logistic regression. 


\section{RESULTS}

Of the 724 patients who began IVF treatment in the Cochin or ClermontFerrand centers in 1998, 293 (40\%) patients obtained a live birth in the inclusion center, 421 (58\%) patients stopped treatment in the center without a live birth and 10 (2\%) patients were either still treated in the center or had an incomplete medical record which made it impossible to determine their treatment outcome (Table 1).

Of the 421 patients who discontinued IVF treatment, 420 were included in the long-term survey (one patient was deceased). Of these, 231 (55\%) patients were treated at Cochin and 189 (45\%) at Clermont-Ferrand. One hundred twenty-three (29\%) patients completed the postal questionnaire, 93 (22\%) patients responded to the brief phone questionnaire and 204 (49\%) patients were non-respondents. Most non-respondents $(n=172,84 \%)$ were patients who could not be either traced or contacted by telephone.

In order to investigate potential bias due to non-response, we compared the three categories of women included in the long-term survey (Table 2): women who responded to the postal questionnaire, women who responded to the phone questionnaire and women who did not respond. They did not significantly differ in age and infertility diagnosis. Non-respondents were more frequent in the Cochin center, if less than 4 oocyte retrievals had been performed and if a long time (6 years or more) had elapsed since discontinuation of IVF in the inclusion center. Among respondents, those who had answered the postal questionnaire were compared with those who answered the short phone questionnaire. We found no difference in patient's profession. Self-reported psychological difficulties still persisting after discontinuation 
of unsuccessful IVF treatment were nearly twice as high among respondents to the short phone questionnaire $(26 \%$ versus $14 \%, p=0.03)$. A slightly higher percentage of postal questionnaire respondents than phone questionnaire respondents had achieved their parenthood project (46\% versus $41 \%$ ) or had a spontaneous pregnancy (21\% versus $16 \%$ ). However, the differences were not statistically significant.

Taken as a whole, of the 216 respondents to the postal or phone questionnaire, 95 (44\%) declared they had achieved their parenthood project since discontinuing unsuccessful IVF treatment in the inclusion center, including 41 (19\%) who had conceived spontaneously. For the 204 patients who did not participate in the long-term survey (non-respondents), various hypotheses can be advanced as to their long-term outcome.

Hypothesis $n^{\circ 1}$ (the extremely pessimistic hypothesis): none of the 204 patients who did not participate in the long-term survey had achieved her parenthood project. This would mean that only 95 patients achieved their parenthood project, representing $23 \%$ of the patients who discontinued unsuccessful IVF treatment and $13 \%$ of the initial 724 patients who began IVF treatment in 1998.

Hypothesis $n^{\circ} 2$ (the intermediate hypothesis): the 204 non-participant patients achieved their parenthood project in the same proportion as respondents to the postal or phone questionnaire (44\%). This would give $185(=95+204 \times 0.44)$ patients who achieved their parenthood project after discontinuation of unsuccessful IVF treatment, representing $44 \%$ of the unsuccessful IVF patients and $26 \%$ of the initial patients. 
Hypothesis $n^{\circ} 3$ (the extremely optimistic hypothesis): all the 204 nonparticipant patients achieved their parenthood project. This would give 299 $(=95+204)$ patients who achieved their parenthood project after discontinuation of unsuccessful IVF treatment, representing $71 \%$ of the unsuccessful IVF patients and $41 \%$ of the initial patients.

Adding the $40 \%$ of patients who had a live birth during IVF treatment, we estimated that the proportion of patients who finally succeeded in their parenthood project during or after IVF treatment was 53\% (pessimistic hypothesis), $66 \%$ (intermediate hypothesis) and 81\% (optimistic hypothesis).

Among respondents to the postal questionnaire $(n=123)$, we were able to explore long-term outcome in greater depth (Table 3). $46 \%$ had achieved their parenthood project, $23 \%$ had not but were still trying to have a child and $31 \%$ had given up their parenthood project. $17 \%$ no longer lived with their male partner of 1998. After discontinuing treatment in their IVF center, patients took different steps to achieve their parenthood project (sometimes with a new male partner): adoption procedure $(42 \%)$, IVF treatment in another center $(20 \%)$, or other medical treatment, in particular artificial insemination with husband or donor sperm (7\%). Among women who achieved their parenthood project after discontinuing unsuccessful treatment, two types of parenthood project achievement predominated: adoption (46\%) and spontaneous pregnancy (42\%). Births after medical treatment (including IVF) appeared much less frequent (12\%). One woman succeeded in two different ways: she first adopted a child and then had a spontaneous pregnancy. 
Factors associated with a successful parenthood project are shown in Table 4. In univariate analysis, six variables were significantly associated with parenthood project achievement. Success was more frequent if the patient was aged less than 35 years at the first oocyte retrieval, if her educational level was medium or high, if she had no child when beginning IVF treatment in the inclusion center in 1998, if duration of infertility in 1998 was less than 5 years, if the decision to discontinue IVF treatment was not voluntary (for example because the treatment was no longer reimbursed) and if in 2006 the patient still lived with the same male partner as in 1998. In multivariate analysis, only three variables remained significant (having no child when beginning IVF treatment, duration of infertility and still living with the same partner). 


\section{DISCUSSION}

Among 724 patients undergoing a first oocyte retrieval in 1998 in one of the two French IVF centers, we estimated, using an intermediate hypothesis, that $66 \%$ of patients finally succeeded in having a child during or after IVF treatment. If we considered extremely pessimistic and optimistic hypotheses (all patients lost to follow-up failed or all succeeded), we concluded that a minimum of $53 \%$ and a maximum of $81 \%$ of patients finally succeeded in their parenthood project. These percentages could continue to rise as more time elapsed since treatment initiation. Indeed, patients could still be attempting to achieve their parenthood project at the time of the survey (as were $23 \%$ of respondents to the postal questionnaire).

According to the intermediate hypothesis (66\% of patients finally succeeding in having a child during or after IVF treatment), the parenthood project was achieved by IVF treatment in the inclusion center $(40 \%)$, by adoption of a child $(12 \%)$, spontaneous pregnancy leading to a live birth $(11 \%)$ or other medical treatment or IVF treatment in another center after discontinuation of unsuccessful treatment in the inclusion center (3\%).

Our results obtained in a French population exhibited the same trends as those observed recently in a Norwegian population. Analyzing long-term outcome of 108 patients referred to one Norwegian IVF center in 1996 and interviewed 10 years later, Sundby et al. found an overall success probability of $77 \%$, including $47 \%$ of patients who had a child during IVF treatment, 15\% who adopted a child and $15 \%$ who had a child following a spontaneous pregnancy (13). In this Norwegian study, 
the authors also reported that $13 \%$ of patients were bringing up a child $(10 \%$ as a foster parent and 3\% as a stepmother).

In our study, we had information on only half of the patients having discontinued IVF without a live birth in the inclusion center. However this low response rate was not linked to refusal to participate: nearly all patients agreed to respond at least to a very short phone questionnaire and $84 \%$ of non-respondents could in fact not be contacted because they had moved since treatment. This is reassuring inasmuch as a high refusal rate often introduces selection bias. Moreover, in our study, non-response was clearly linked with variables that could be associated with a higher risk of a change of address: treatment in the Paris region (Cochin center) where the population is more mobile than in other regions of France (such as Clermont-Ferrand), a longer time elapsing since IVF discontinuation and having less than four oocyte retrievals, which is linked with time elapsed since IVF. The rate of follow-up loss observed in our study $(49 \%)$ was close to that $(42 \%)$ observed in a Brazilian study based on phone interviews of 92 couples who had unsuccessfully undergone one or more IVF cycles in an IVF center at least 3 years before the start of the study (14). Such a high rate of follow-up loss in our study was due to the long time elapsing between IVF treatment discontinuation and our survey, more than 6 years for $89 \%$ of non-respondents versus $79-82 \%$ of respondents $(p=0.04)$. Such a long period was necessary to investigate long-term outcome (especially adoption, a procedure which requires several years). Another methodological approach could be to consider active follow-up of a cohort of patients. However, this approach is very time-consuming and costly and could finally be inefficient. Firstly, the topic of our survey is a very sensitive one for patients and questionnaires repeated over time could be difficult to administer. Secondly, loss of follow-up in a cohort is commonly as 
high as $40-50 \%$ after 6 years. For example, in a French cohort on contraception, of the 2,863 women who agreed in 2000 to participate in a yearly phone contact, $33 \%$ no longer participated only two years later (15) and about $20 \%$ of UK families who participated in the first sweep of the Millennium Cohort Study did not respond to the second sweep just two years later (16). When investigating long-term parenthood projects among unsuccessful IVF treatment patients, which is a very sensitive topic for those who have not yet succeeded, one could expect an even higher rate of follow-up loss if carrying out repeated distressing interrogations in a prospective cohort.

A high rate of non-response could lead to selection bias in our study if reasons for moving were related to the patient's probability of achieving her parenthood project. We first considered that the change of residence could be related to separation of the couple, which was a major predictive factor of failure to have a child after IVF treatment discontinuation among respondents. So, non-respondents could have a lower chance of success than respondents. We also considered that, on the contrary, change of residence could be related to the arrival of a child in the family and the need for an additional bedroom (17). In view of these two contradictory assumptions, we were unable to conclude on the direction selection bias would go, whether it would imply over- or under-estimation of the probability of finally having a child. Finally, two methods of interviewing patients were used in the study (postal questionnaire and phone interview). We do not believe that this would induce differential bias in factual information such as the birth or adoption of a child. However, it could have induced differences for less factual information such as psychological well-being. In Table 2, we observed that patients responding to the short phone questionnaire were twice as likely to declare psychological problems 
than respondents to the postal questionnaire. Psychological difficulties could be the reason why these patients did not answer the postal questionnaire, but we could not exclude that this partly reflected differences linked to the method of interview (phone or postal questionnaire).

In our multivariate analysis, achievement of the parenthood project after discontinuation of unsuccessful IVF treatment in the inclusion center was positively linked to two initial characteristics of the patient: not having a child at the beginning of IVF treatment and infertility duration of less than 5 years in 1998. Both could reflect patients' motivation and determination to succeed in having a child (even by adoption) and/or better fertility chances leading to spontaneous pregnancies (patients with shorter infertility duration possibly being more fertile and also younger). Two other variables could be interpreted in the same way: young age of the patient at the first oocyte retrieval and the fact that discontinuation of IVF treatment was not a voluntary decision by the couple. However, these two variables were significant only in univariate analysis. Moreover, patients separated from their male partner appeared to have less chance of finally having a child, probably because separation could have put an end to the parenthood project and/or because the patient was then alone. This suggests that the long-term outcome of a parenthood project may vary from one country to another according to the frequency of separations. For example, in France, the divorce rate was $42.4 \%$ in 2003 , while it was higher $(56.1 \%)$ in Belgium and very much lower in Spain (14.5\%) (18). Similar research on the long-term outcome of parenthood project should be carried out in other countries in order to verify that the observations made in French couples can be generalized. 
Our results showed the importance of spontaneous pregnancy and of adoption in achieving the parenthood project after discontinuation of unsuccessful IVF treatment, whereas only a small proportion of patients became parents following another medical treatment. Surprisingly, although $20 \%$ of patients followed another IVF program in another center, only $21 \%$ of these patients had a child in this way. In our survey, $19 \%$ of respondents reported that they had spontaneously conceived since discontinuation of IVF. This proportion is similar to those reported in two studies analyzing spontaneous pregnancy rate among IVF patients who had a live birth after treatment: a Japanese study observed that $18 \%$ of 142 patients conceived spontaneously within 5 years after the birth of their IVF child (19) and an Irish study found that $21 \%$ of 513 patients conceived spontaneously after an IVF birth (20). In a Belgian study, spontaneous pregnancies were analyzed among 200 patients discontinuing ICSI (21). The authors observed a slightly lower rate of spontaneous pregnancy, $12-13 \%$, but the time since treatment discontinuation was shorter (3 years).

Our long-term survey showed that adoption and spontaneous conception are important issues to consider when dealing with the long-term outcome of parenthood projects among couples beginning an IVF program. It would be very interesting to examine in greater depth which patients finally conceived spontaneously and which patients finally adopted a child. This approach was not possible in our study because the sample size was too small, so more research would be needed on this topic.

In conclusion, we gained new insight for IVF patients by taking a long-term view rather than considering their parenthood project only in the IVF center. While for 
$40 \%$ of patients IVF treatment resulted in a successful pregnancy, unsuccessful patients should not lose hope, as nearly half of them may subsequently have a child. 


\section{REFERENCES}

1. Boivin J, Bunting L, Collins JA, Nygren KG. International estimates of infertility prevalence and treatment-seeking: potential need and demand for infertility medical care. Hum Reprod 2007;22:1506-12.

2. Nyboe Andersen A, Goossens V, Gianaroli L, Felberbaum R, de Mouzon J, Nygren KG. Assisted reproductive technology in Europe, 2003. Results generated from European registers by ESHRE. Hum Reprod 2007;22:151325.

3. Eugster A, Vingerhoets AJ. Psychological aspects of in vitro fertilization: a review. Soc Sci Med 1999;48:575-89.

4. Schmidt L. Psychosocial burden of infertility and assisted reproduction. Lancet 2006;367:379-80.

5. Land JA, Courtar DA, Evers JL. Patient dropout in an assisted reproductive technology program: implications for pregnancy rates. Fertil Steril 1997;68:278-81.

6. Olivius K, Friden B, Lundin K, Bergh C. Cumulative probability of live birth after three in vitro fertilization/intracytoplasmic sperm injection cycles. Fertil Steril 2002;77:505-10.

7. Sharma V, Allgar V, Rajkhowa M. Factors influencing the cumulative conception rate and discontinuation of in vitro fertilization treatment for infertility. Fertil Steril 2002;78:40-6.

8. Domar AD. Impact of psychological factors on dropout rates in insured infertility patients. Fertil Steril 2004;81:271-3.

9. Olivius C, Friden B, Borg G, Bergh C. Why do couples discontinue in vitro fertilization treatment? a cohort study. Fertil Steril 2004;81:258-61. 
10. Penzias AS. When and why does the dream die? or does it? Fertil Steril 2004;81:274-5.

11. Soullier N, Bouyer J, Pouly JL, Guibert J, de La Rochebrochard E. Estimating the success of an in vitro fertilization programme using multiple imputation. Hum Reprod 2008;23:187-92.

12. Stolwijk AM, Hamilton CJ, Hollanders JM, Bastiaans LA, Zielhuis GA. A more realistic approach to the cumulative pregnancy rate after in-vitro fertilization. Hum Reprod 1996;11:660-3.

13. Sundby J, Schmidt L, Heldaas K, Bugge S, Tanbo T. Consequences of IVF among women: 10 years post-treatment. J Psychosom Obstet Gynaecol $2007 ; 28: 115-20$.

14. Filetto JN, Makuch MY. Long-term follow-up of women and men after unsuccessful IVF. Reprod Biomed Online 2005;11:458-63.

15. Razafindratsima N, Kishimba N, COCON Group. Attrition in the COCON cohort between 2000 and 2002. Popul (English) 2004;59:357-85.

16. Plewis I, Ketende S. Millennium Cohort Study: Technical Report on Response. London: Centre for Longitudinal Studies, Institute of Education; 2006.

17. Courgeau D, Lelievre E. Estimation des migrations internes de la période 1990-1999 et comparaison avec celles des périodes antérieures. Popul 2004;59:797-804.

18. Sardon JP. Recent demographic trends in developed countries. Popul (English) 2006;61:197-266.

19. Shimizu Y, Kodama H, Fukuda J, Murata M, Kumagai J, Tanaka T. Spontaneous conception after the birth of infants conceived through in vitro fertilization treatment. Fertil Steril 1999;71:35-9. 
20. Hennelly B, Harrison RF, Kelly J, Jacob S, Barrett T. Spontaneous conception after a successful attempt at in vitro fertilization/intracytoplasmic sperm injection. Fertil Steril 2000;73:774-8.

21. Osmanagaoglu K, Collins J, Kolibianakis E, Tournaye H, Camus M, Van Steirteghem A, et al. Spontaneous pregnancies in couples who discontinued intracytoplasmic sperm injection treatment: a 5-year follow-up study. Fertil Steril 2002;78:550-6. 
TABLE 1. Treatment outcomes among the 724 patients who began IVF treatment in 1998 in one of the two IVF centers.

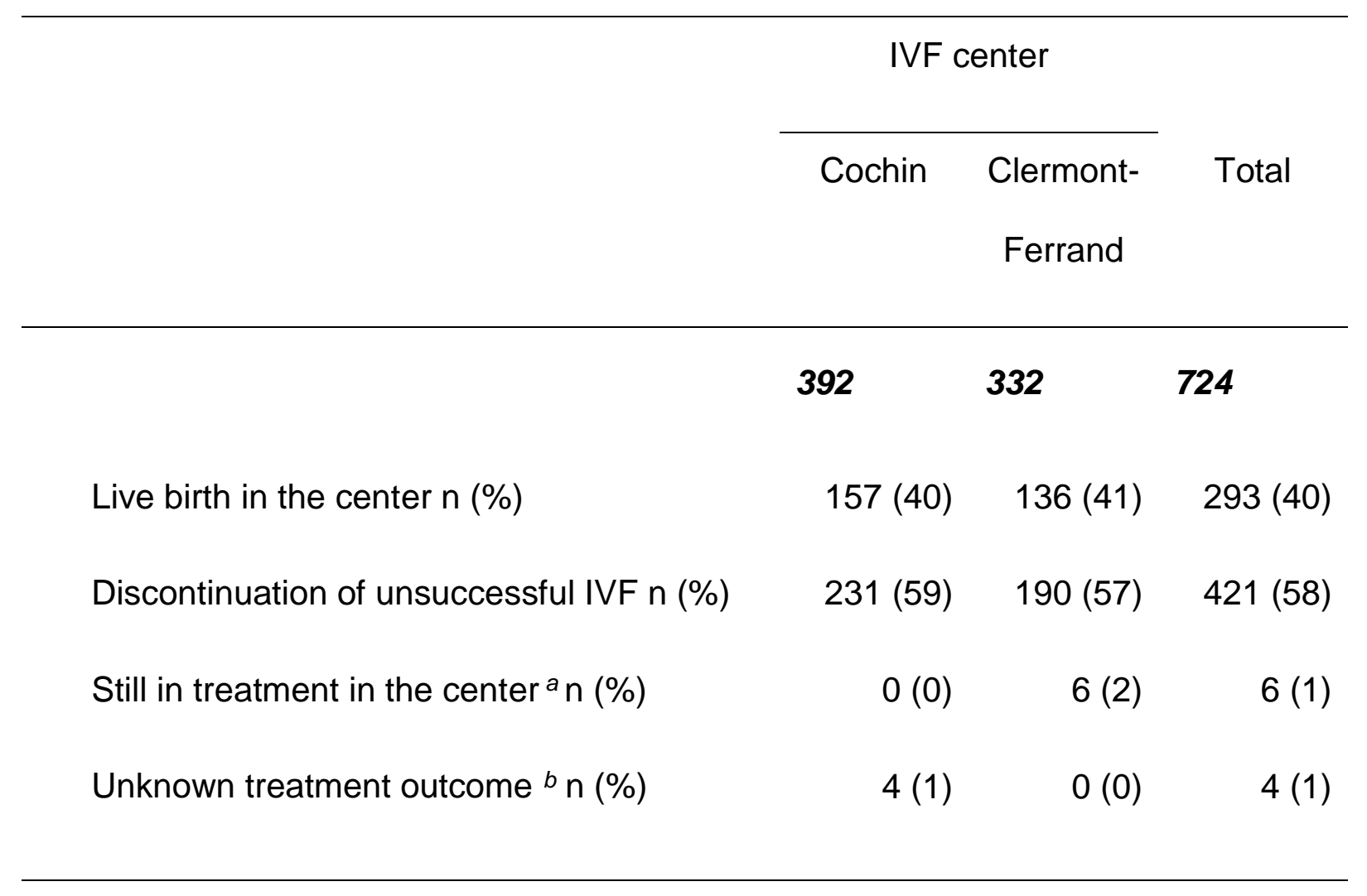

a: We considered that a patient was still in treatment in the inclusion center if she had an oocyte retrieval or a fresh or frozen embryo transfer or an IVF pregnancy in the center in the two years before our mailed questionnaire in May 2006.

b: Outcome of IVF treatment was unknown for 4 patients in the Cochin center because the information was missing from the medical records. 
TABLE 2. Characteristics (\%) of postal questionnaire respondents, short phone questionnaire respondents, and non-respondents $(n=420$ a patients included in the long-term survey).

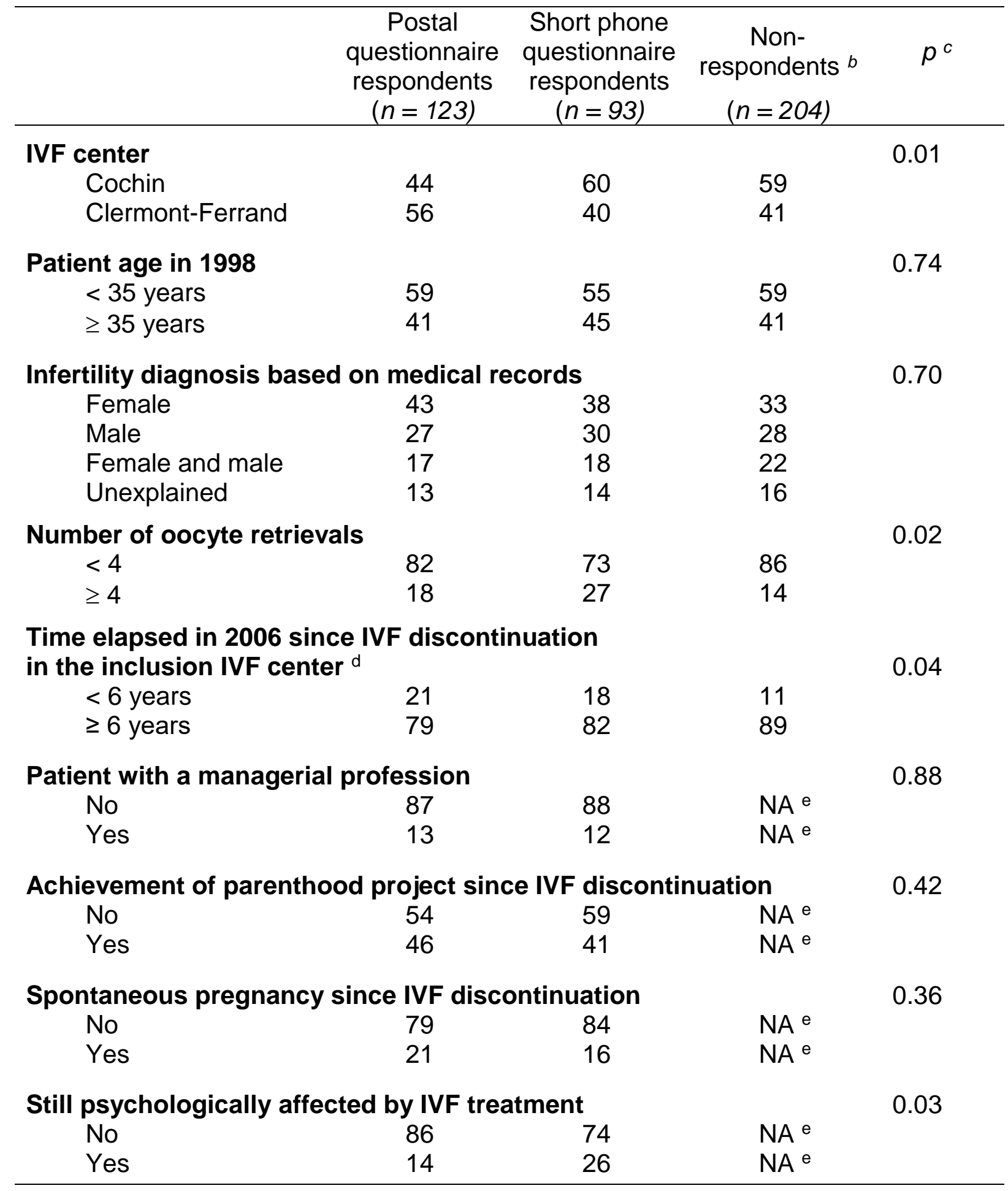

a: Of the 421 patients who discontinued unsuccessful IVF treatment, one was deceased.

$b$ : Non-respondents include patients lost to follow-up $(n=178)$ and patients refusing to answer $(n=26)$.

$c: \quad p$ value of the chi-squared or Fisher exact test.

$d$ : Time elapsed in 2006 since discontinuation of unsuccessful IVF treatment in the inclusion center was from 3 to 8 years. Six years was the first percentile (median: 7 years, $3^{\text {rd }}$ 
percentile: 8 years).

e: $\mathrm{NA}=$ not applicable: this information was not available for non-respondents. 
TABLE 3. Long-term parenthood project outcome after discontinuation of unsuccessful IVF treatment in the inclusion center among the 123 respondents to the postal questionnaire in the long-term survey.

Long-term outcome of the patient's parenthood project

Succeeded in her parenthood project

Project continuing

Project abandoned

$31 \%$

Situation of the couple since IVF discontinuation

Still living together

$83 \%$

Separated

$17 \%$

Steps taken to achieve (successfully or not) the parenthood project $^{a}$

IVF treatment in another center $20 \%$

Medical treatment other than IVF

Start of adoption procedure $42 \%$

For patients with a successful parenthood project $(n=57)$, method by which it had been achieved $b$

IVF treatment in another center

Other medical treatment (including Al with donor $\quad 5 \%$

sperm)

Adoption

Spontaneous pregnancy leading to a live birth $\quad 42 \%$

a: One patient may have taken none, one or more than one steps, so the sum of percentages is not $100 \%$.

$b$ : If the patient had more than one child, we only considered the first, so the sum of column percentages is $100 \%$. 
TABLE 4. Factors associated with achievement of parenthood project after discontinuation of unsuccessful IVF treatment in the inclusion center among the 123 respondents to the postal questionnaire.

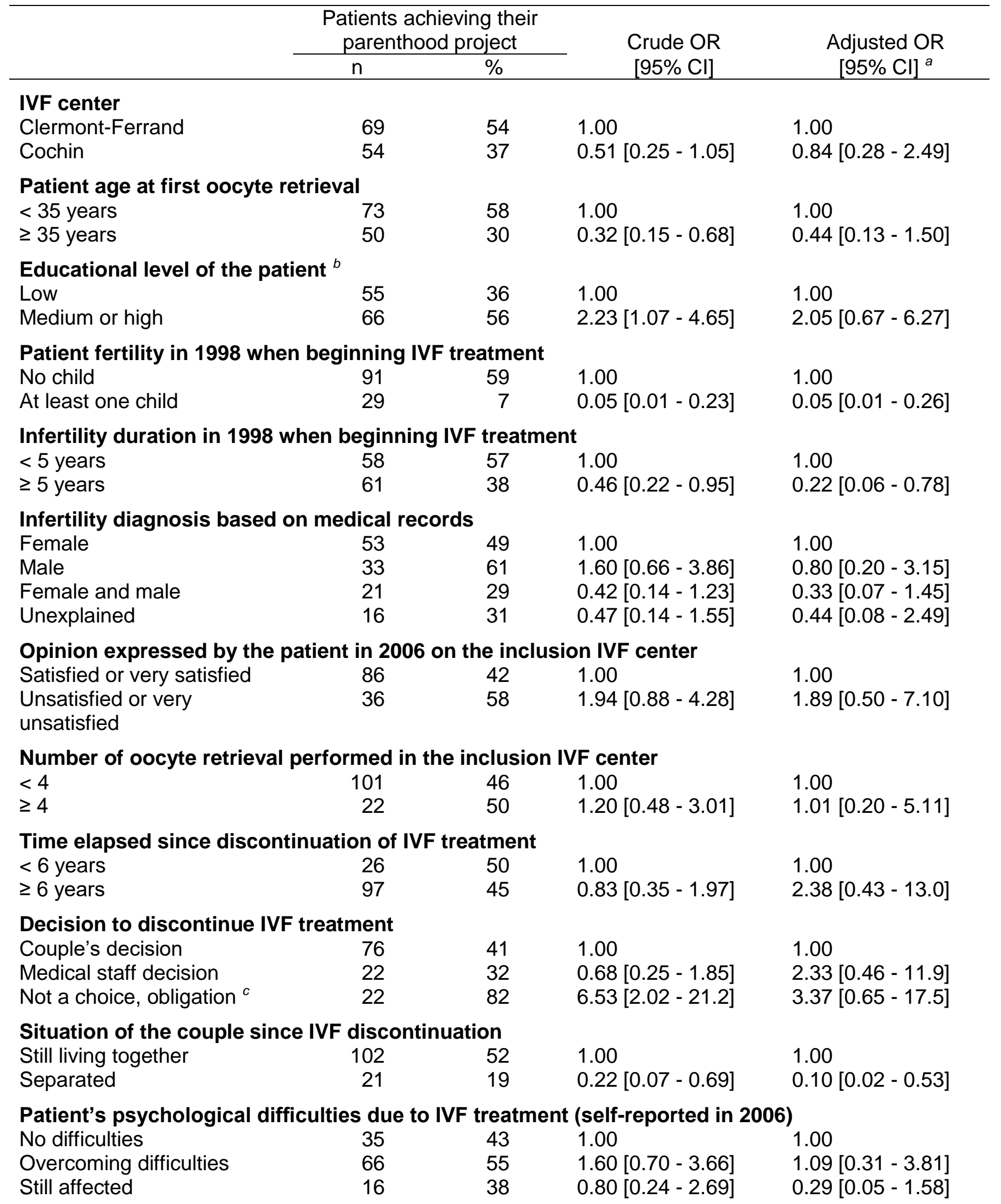


a: $\mathrm{n}=107$ patients of 123 with complete information for the 12 variables entered in the multivariate model.

$b$ : Low level of education was defined as < baccalauréat; medium-high level was defined as $\geq$ baccalauréat (completion of secondary education).

c: Main reasons for considering that IVF discontinuation was not a choice but was involuntary were that the treatment was no longer reimbursed or that the patient was too old to be included in an IVF treatment program. 